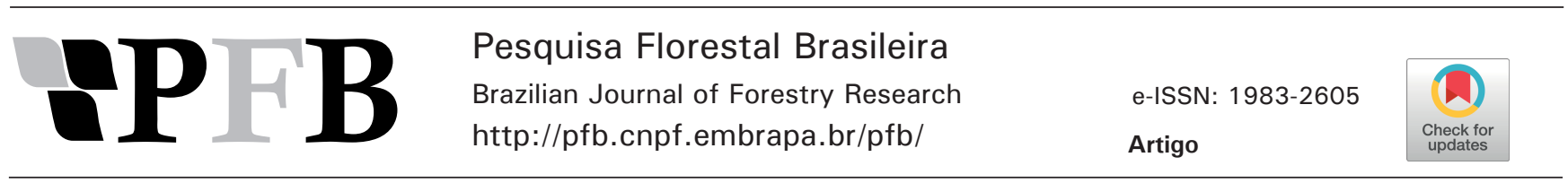

\title{
Morfometria de Araucaria angustifolia em diferentes altitudes no Sul do Brasil
}

\author{
Pollyni Ricken ${ }^{1}$ (D), André Felipe Hess ${ }^{2}$ (D), Patrícia Póvoa de Mattos ${ }^{3}$ (D), Evaldo Muñoz Braz ${ }^{3}$ (D), Nelson Yoshihiro Nakajima ${ }^{1}$ (D), \\ Roberto Tuyoshi Hosokawa ${ }^{1}$
}

\author{
'Universidade Federal do Paraná, Avenida Prefeito Lothário Meissner, 3400, Jardim Botânico, CEP 80210-170, Curitiba, PR, Brasil \\ ${ }^{2}$ Universidade do Estado de Santa Catarina, Av. Luiz de Camões, 2090, Conta Dinheiro, CEP 88520-000, Lages, SC, Brasil \\ ${ }^{3}$ Embrapa Florestas, Estrada da Ribeira, km 111, C.P. 319, CEP 83411-000, Colombo, PR, Brasil
}

\section{"Autor correspondente:}

pollyni7@hotmail.com

Termos para indexação:

Análise de crescimento

Floresta com Araucária

Método de Bitterlich

Index terms:

Growth analysis

Araucaria Forest

Bitterlich method

Histórico do artigo:

Recebido em 21/11/2019

Aprovado em 13/02/2020

Publicado em 12/05/2020

\section{c) $(1)$}

Resumo - Os índices morfométricos podem ser usados para subsidiar o entendimento da dinâmica de florestas naturais. Diante disso, objetivou-se descrever e avaliar as características morfométricas e dendrométricas de Araucaria angustifolia, como subsídio para compreensão da estrutura da floresta natural de araucária. Os dados foram mensurados em três municípios de Santa Catarina, considerando diferentes altitudes de ocorrência da espécie, com amostragem de 247 árvores, utilizando o método de Bitterlich, sendo medida a circunferência a $1,30 \mathrm{~m}$ do solo, altura total e de inserção de copa e raios de copa. As relações interdimensionais das árvores foram descritas por meio de índices morfométricos. O sítio 2 apresentou árvores de maior porte em relação ao diâmetro e área de copa. O sítio 3 apresentou a maior densidade de árvores (403 árvores ha-1), enquanto nos sítios 1 e 2 foram observadas 362 e 232 árvores ha-1, respectivamente. No sítio 3, a maior parte das árvores apresentou proporção de copa superior a $50 \%$ e formal de copa menor que 1 , indicando árvores de copas mais estreitas e largas. O diâmetro da árvore apresentou correlação negativa e superior a 70\% com o grau de esbeltez. Nos três sítios, o diâmetro apresentou correlação positiva com o diâmetro de copa.

\section{Morphometry of Araucaria angustifolia at different altitudes in Southern Brazil}

\begin{abstract}
Morphometric indices can be used to support the understanding of natural forest dynamics. The aim of this study was to describe and evaluate the morphometric and dendrometric characteristics of Araucaria angustifolia, to understanding of the structure of natural Araucaria Forest. The data were obtained in three counties of Santa Catarina State, considering different altitudes of occurrence of the species. We sampled 247 trees, using the Bitterlich method, and we measured the circumferences at $1.30 \mathrm{~m}$ from the ground, total height and crown insertion height and crown radius. The trees interdimensional relationships were described through morphometric indices. Site 2 showed larger trees considering crown area diameter. Site 3 had the highest tree density $\left(403\right.$ trees ha ${ }^{-1}$ ), while sites 1 and 2 showed 362 and 232 trees ha ${ }^{-1}$, respectively. At site 3 , most trees presented crown ratio greater than $50 \%$ and crown fullness ratio lower than 1, indicating trees with narrower and larger crowns. The tree diameter showed a negative correlation, higher than $70 \%$ with the degree of slenderness. The diameter presented a positive correlation with the crown diameter in the three sites.
\end{abstract}




\section{Introdução}

Araucaria angustifolia (Bert.) O. Kuntze é o principal componente arbóreo do estrato da Floresta Ombrófila Mista (FOM) ou Floresta com Araucárias (Valério et al., 2017), tendo essas matas com araucária ocupado, no passado, 20 milhões de ha da paisagem no Sul do Brasil. No entanto, a crescente necessidade de terras para a agricultura e pecuária bem como as amplas possibilidades de uso de sua madeira acarretou, desde a metade do século passado, a exploração dessas matas (Souza et al., 2015). De acordo com Machado et al. (2008), outras espécies da Floresta Ombrófila Mista, tais como Ocotea porosa (Mez) L. Barroso e Cedrela fissilis Vell., também sofreram com a exploração de recursos madeiráveis e com o processo de colonização e expansão das áreas agrícolas.

Segundo Leite \& Klein (1990), a tipologia da região da Floresta Ombrófila Mista foi drasticamente reduzida a inexpressivos $10 \%$ de sua dimensão original, antes mesmo de ser adequadamente conhecida.

A redução do tamanho e do número de remanescentes da FOM tem resultado em degradação das populações de araucária. Em alguns casos, essas populações tornam-se vulneráveis, em resposta ao rompimento de sua dinâmica de regeneração, amadurecimento e reprodução (Shimizu et al., 2000).

Araucaria angustifolia ocorre de forma natural em regiões serranas e planaltos, situando-se numa altitude superior a $400 \mathrm{~m}$, podendo chegar a $2.300 \mathrm{~m}$ de altitude (Mähler Junior \& Larocca, 2009; Mattos, 2011). Sua ocorrência natural está relacionada às condições climáticas, pois, quanto mais elevada a altitude, mais baixas são as temperaturas médias e extremas. A espécie é resistente à geada e à seca e, embora não seja o seu ambiente ideal, tolera climas quentes. Tolera também umidade relativa alta, porém não se desenvolve bem em solos com umidade elevada (Mattos, 2011).

Informações tais como o número de árvores, a localização da árvore, altura, e diâmetro da copa são essenciais para a análise quantitativa da floresta (Chen et al., 2006; Koch et al., 2006; Kwak et al., 2007). Diversos índices obtidos entre as dimensões da copa e do tronco buscam descrever a capacidade de crescimento e produção das árvores e da floresta (Padoin \& Finger, 2010).

O estudo da morfometria da copa das árvores permite entender as relações interdimensionais e reconstruir o espaço utilizado por elas. Também é possível avaliar o grau de competição que determinado povoamento está submetido e inferir sobre o desenvolvimento das árvores (Durlo \& Denardi, 1998). Assim, podem subsidiar informações relevantes, em conjunto com outros indicadores de condições da estrutura da floresta, para a determinação de práticas de intervenção silvicultural (Silva et al., 2017).

Uma das relações mais utilizadas para a análise do desenvolvimento das plantas é o grau de esbeltez (ge), que é a relação entre a altura total (h) e o diâmetro a 1,30 m de altura do solo (dap). Tonini \& ArcoVerde (2005) afirmam que uma relação h/dap maior que 1 (um) indica a necessidade de desbaste.

O diâmetro da copa ( $\mathrm{dc}$ ) é a variável básica para se estimar as demais características da árvore, correspondendo ao comprimento da linha de projeção entre os pontos extremos da copa. Outros parâmetros usados são o índice de saliência (is), que relaciona o diâmetro da copa e o dap, e a razão entre o diâmetro da copa e a altura total da árvore, conhecida como índice de abrangência (ia) (Durlo \& Denardi, 1998). Tonini \& ArcoVerde (2005) relatam que este índice tende a diminuir com a altura, já que o crescimento em altura da árvore não é acompanhado, proporcionalmente, pelo crescimento em diâmetro da copa.

Outro índice utilizado para avaliar a forma geométrica da copa é o formal de copa (fc), que expressa a relação entre o diâmetro e o comprimento da copa. Roman et al. (2009) citam que valores baixos de formal de copa indicam copas mais esbeltas enquanto valores maiores definem copas mais arredondadas.

A estimativa dos parâmetros morfométricos podem ser usados para compreender a estrutura da floresta, onde a retirada de indivíduos propiciaria um aumento na incidência de luz, além de diminuir a concorrência por espaço e nutrientes entre os indivíduos, favorecendo o crescimento dos remanescentes.

Os objetivos deste trabalho foram avaliar e relacionar as variáveis morfométricas e dendrométricas de Araucaria angustifolia em três altitudes de ocorrência natural, e inferir sobre a densidade de árvores por hectare, visando a otimização do desenvolvimento das árvores.

\section{Material e métodos}

O estudo foi realizado em áreas de floresta natural de Araucaria angustifolia localizadas em três municípios de Santa Catarina. Os locais de estudo foram: Bom Jardim 
da Serra (sítio 1, altitude média de $1.350 \mathrm{~m}, 28^{\circ} 20^{\prime} 31^{\prime \prime} \mathrm{S}$ e $49^{\circ} 35^{\prime} 10^{\prime \prime} \mathrm{W}$, precipitação pluviométrica média anual de $1.670 \mathrm{~mm}$ ), Painel (sítio 2, altitude média de $1.150 \mathrm{~m}$, $27^{\circ} 53^{\prime} 57^{\prime}$ 'S e $50^{\circ} 07^{\prime} 42^{\prime \prime} \mathrm{W}$, precipitação pluviométrica média anual de $1.125 \mathrm{~mm}$ ) e São José do Cerrito (sítio 3, altitude média de $950 \mathrm{~m}, 27^{\circ} 42^{\prime} 40^{\prime \prime} \mathrm{S}$ e $50^{\circ} 28^{\prime} 51^{\prime \prime} \mathrm{W}$, precipitação pluviométrica média anual de $1.570 \mathrm{~mm}$ ). Os dados de precipitação pluviométrica média anual foram obtidos da Epagri (2002).

O clima nas três áreas é caracterizado, segundo Köppen, como sendo predominantemente do tipo $\mathrm{Cfb}$ temperado propriamente dito, com temperatura média do mês mais frio abaixo de $18{ }^{\circ} \mathrm{C}$ (mesotérmico), com possibilidade de geadas (Epagri, 2002).

As árvores para coleta de dados foram selecionadas por processo de amostragem aleatório, empregando-se o método de Bitterlich, com fator de área basal (FAB) 2. Foram amostradas 77, 93 e 77 árvores, nos locais 1, 2 e 3, respectivamente, totalizando 247 árvores. A suficiência amostral indicou representativo o número de pontos em cada local de amostragem, que foram 7 em Bom Jardim da Serra, 6 em Painel e 6 em São José do Cerrito.

Foram realizadas as seguintes medições em todas as árvores: (i) circunferência a 1,30 m do solo (cap); (ii) altura total (h) e de inserção de copa (hi), medidas com auxílio do aparelho Trupulse; (iii) raios de copa: medidos com uma bússola e com auxílio do Trupulse, em quatro direções dos pontos cardeais, com intervalo de 90 graus $(\mathrm{N}, \mathrm{S}, \mathrm{L}$ e W), sendo a média aritmética dos quatro raios determinada como raio de copa (em $\mathrm{m}$ ).

$\mathrm{O}$ número de árvores por hectare $(\mathrm{N})$ e a área basal (G) foram estimados conforme o método de Bitterlich, usando as equações 1 e 2, apresentadas por Soares et al. (2011).

$$
N=F A B * \sum\left(1 / g_{1}\right)
$$

Onde: $N=$ número de árvores ha ${ }^{-1} ; F A B=$ Fator de área basal; e $g_{l}=$ área transversal.

$$
G=m * F A B
$$

Onde: $G=$ Área basal $\mathrm{em} \mathrm{m}^{2} \mathrm{ha}^{-1} ; m=$ número de árvores incluídas no ponto amostral; e $F A B=$ Fator de área basal.

Os cálculos foram realizados para cada parcela individualmente e, posteriormente, obtidas as médias para cada local.

Para descrever as relações interdimensionais das árvores, foram obtidas as seguintes variáveis morfométricas: (i) área de projeção de copa (ac): utilizando a média dos quatro raios de copa ao quadrado, multiplicando pelo valor de pi e, ao final, dividindo-a por 4; (ii) proporção de copa (\%) ou percentagem de copa (pc): relação entre o comprimento da copa e a altura total da árvore; (iii) formal de copa (fc): razão entre o diâmetro de copa e o comprimento de copa; (iv) grau de esbeltez (ge): razão entre a altura total das árvores e o diâmetro a 1,30 m de altura do solo; (v) índice de abrangência (ia): razão entre o diâmetro da copa e a altura total; e (vi) índice de saliência (is): razão entre o diâmetro da copa e o diâmetro a 1,30 m de altura do solo.

As correlações de Pearson foram estabelecidas entre as variáveis morfométricas e dendrométricas avaliadas, com nível de significância de 95\%. A significância da média das variáveis mensuradas entre locais foi analisada por meio do teste de Kruskal-Wallis.

Foram testados 9 modelos para o ajuste das relações h dap ${ }^{-1}$, para cada local (equações 3, 4, 5, 6, 7, 8, 9, 10 e 11), sendo a melhor equação obtida mediante ajustes comparativos dos parâmetros.

$$
\begin{aligned}
& \mathrm{h}=\beta_{0}+\beta_{1}{ }^{*} \text { dap }+\beta_{2}{ }^{*} \text { dap }+\beta_{2}{ }^{*} \text { dap }{ }^{2} \\
& \text { Stofells } \\
& \operatorname{Ln} \mathrm{h}=\beta_{0}+\beta_{1}{ }^{*} \operatorname{Ln} \text { dap } \\
& \text { Curtis } \\
& \operatorname{Ln~} \mathrm{h}=\beta_{0}+\beta_{1}^{*}(1 / \text { dap }) \\
& \text { Curtis } \\
& \mathrm{h}=\beta_{0}+\beta_{1}^{*}(1 / \text { dap }) \\
& \mathrm{h}=\beta_{0}+\beta_{1} * \text { dap }+\beta_{2}^{*}(1 / \text { dap }) \\
& \text { - } \quad \mathrm{h}=\beta_{0}+\beta_{1}^{*}(1 / \mathrm{dap})+\beta_{2}^{*}(1 / \mathrm{dap}) \\
& \text { - } \quad \mathrm{h}=\beta_{0}+\beta_{1} * \text { dap }+\beta_{2}{ }^{*}(1 / \text { dap })+\beta_{3}{ }^{*} \text { dap }{ }^{2} \\
& \text { - } \quad \mathrm{h}=\beta_{0}+\beta_{1}{ }^{*} \text { dap } \\
& \text { - } \quad \mathrm{h}=\beta_{0}+\beta_{1}{ }^{*} \text { dap }+\beta_{2}{ }^{*}(1 / \text { dap })+\beta_{3}{ }^{*} \text { dap }{ }^{3}
\end{aligned}
$$

Onde: dap = diâmetro a $1,30 \mathrm{~m}$ de altura do solo $(\mathrm{cm}) ; \mathrm{h}$ $=$ altura total $(\mathrm{m}) ; \beta_{0}, \ldots, \beta_{3}=$ coeficientes ajustados; $\mathrm{Ln}=$ Logaritmo natural.

A fração de ocupação (F. Ocup.) pela copa foi calculada com base na área de copa para 1 hectare, adaptada de Assmann (1970), conforme equação 12.

$$
\text { F. Ocup. }=\left(N^{*} a c\right) 10.000
$$

Onde: $N=$ número de árvores ha $^{-1}$; e $a c=$ área de projeção de copa média $\left(\mathrm{m}^{2}\right)$.

Para avaliar o grau de ocupação, para cada local, as árvores foram agrupadas de acordo com seus respectivos diâmetros em estrato inferior $(10 \leq$ dap $<40 \mathrm{~cm})$, estrato médio ( $40 \leq$ dap $<60 \mathrm{~cm}$ ) e estrato superior (dap $\geq 60 \mathrm{~cm}$ ) 
(Daniel et al., 1979; Feldpausch et al., 2011; Braz et al., 2015), sendo neste cálculo utilizado os valores médios de área de copa obtidos em cada classe de dap.

\section{Resultados}

No estudo das características dendrométricas e morfométricas de Araucaria angustifolia, foi possível obter as diferentes distribuições diamétricas resultantes de cada um dos locais (Figura 1).
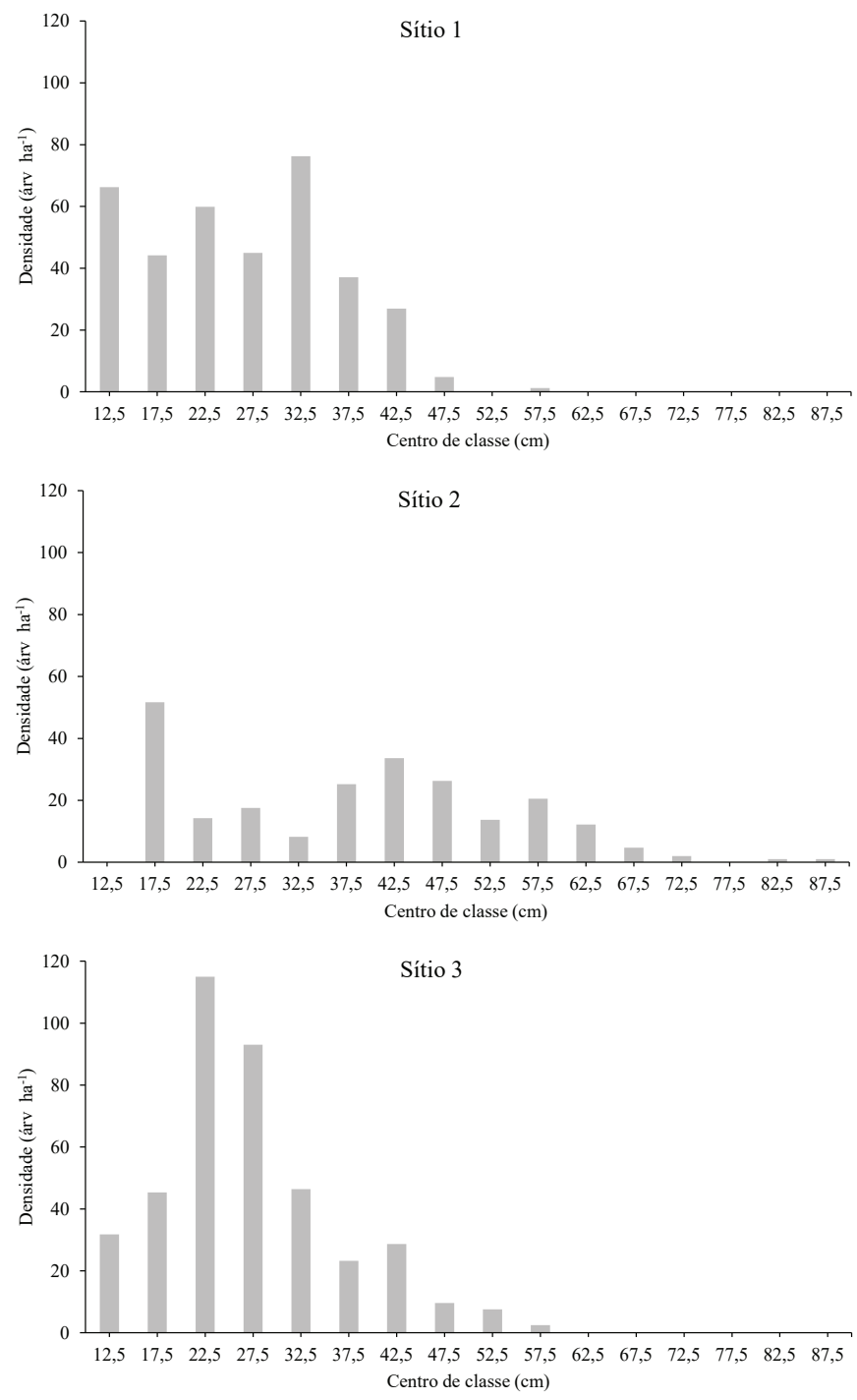

Figura 1. Distribuição diamétrica de Araucaria angustifolia nos três locais, em Santa Catarina, Brasil.

Figure 1. Diametric distribution of Araucaria angustifolia in the three sites in Santa Catarina State, Brazil.
Os diâmetros a 1,30 $\mathrm{m}$ do solo (dap) variaram entre 12,7 e $55,1 \mathrm{~cm}$ (S1), 17,8 e $88,2 \mathrm{~cm}$ (S2) e 11,6 e $59,8 \mathrm{~cm}$ (S3) e as alturas (h) entre 8,0 e 23,2 m (S1), 9,2 e 27,7 m (S2) e 7,2 e 23,7 m (S3). A área basal (G) encontrada para os sítios 1, 2 e 3 foram, respectivamente, 22,0, 31,0 e $25,67 \mathrm{~m}^{2} \mathrm{ha}^{-1}$.

Foi observada diferença em relação aos diâmetros encontrados em cada uma das áreas, sendo que, no sítio 2 , não foram encontrados indivíduos com $10 \mathrm{~cm} \geq$ dap $>15 \mathrm{~cm}$. Os sítios 1 e 3 apresentaram semelhança em relação às classes maiores, sendo registrados indivíduos com dap $>60 \mathrm{~cm}$ apenas no sítio 2 .
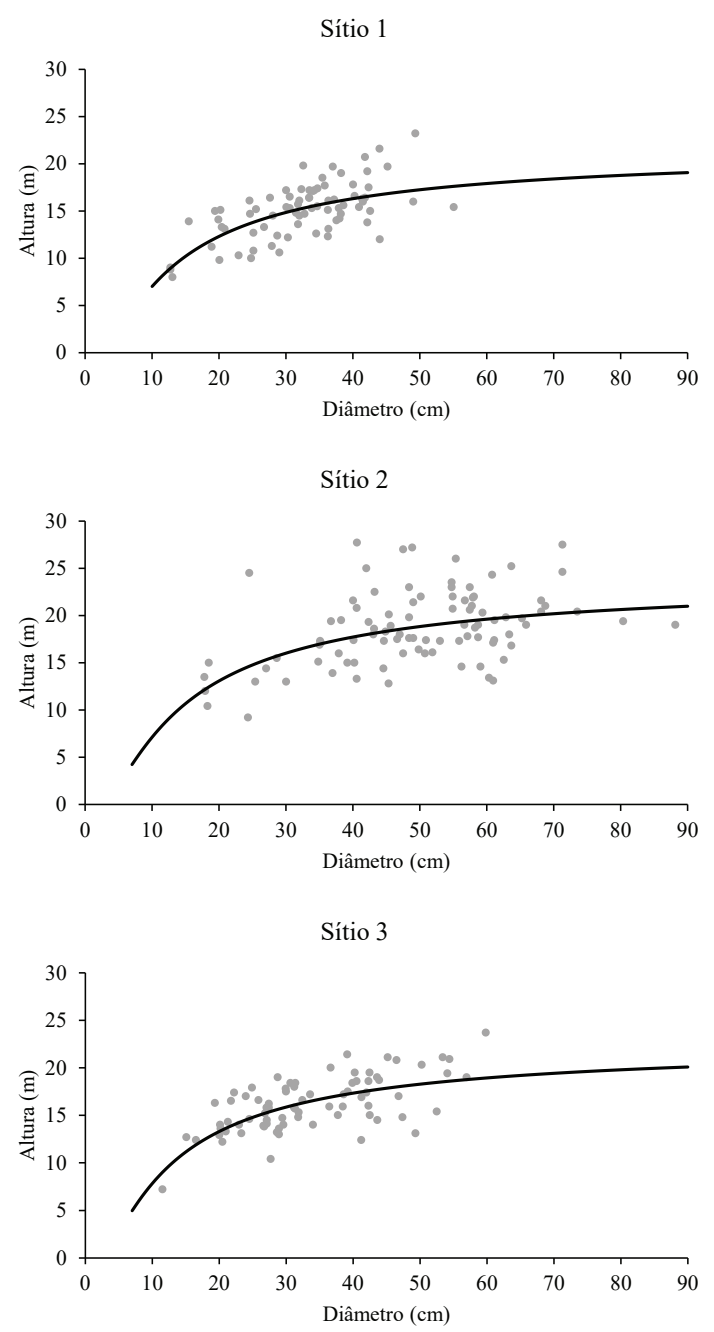

Figura 2. Dados de altura (h) e diâmetro a 1,30 m do solo (dap) e relações h/dap ajustadas de Araucaria angustifolia para três sítios, em Santa Catarina, Brasil.

Figure 2. Height (h) and diameter at $1.3 \mathrm{~m}$ above ground level (dbh) data and adjusted h/dbh ratios of Araucaria angustifolia to three sites in Santa Catarina State, Brazil. 
Os valores de dap e altura mensuradas são apresentados na Figura 2, bem como as relações h/dap ajustadas para cada local.

A melhor equação hipsométrica ajustada para as três áreas foi a de Curtis, sendo os coeficientes e parâmetros apresentados na Tabela 1.

Os parâmetros morfométricos por área estão apresentados na Tabela 2, bem como a análise de significância entre as variáveis mensuradas em campo.
O grau de esbeltez (ge), ou relação altura/diâmetro média, foi semelhante entre os sítios, com variação de 0,4 a 0,5 e uma dispersão homogênea em torno da média $(20 \%$ a $25 \%)$. Apenas uma árvore no sítio 2 teve grau de esbeltez igual a 1.

A proporção de copa (pc), dada pela relação entre comprimento da copa e altura total variou de $17,2 \%$ (sítio 1) a 54,5\% (sítio 3). Apesar do valor médio de pc ser menor no sítio 1, esta área apresentou dispersão em

Tabela 1. Coeficientes e parâmetros da equação hipsométrica de Curtis para Araucaria angustifolia, para três locais, em Santa Catarina, Brasil.

Table 1. Coefficients and parameters for the Curtis hypsometric equation for Araucaria angustifolia in the three sites, in Santa Catarina State, Brazil.

\begin{tabular}{|c|c|c|c|c|c|c|c|}
\hline \multirow{2}{*}{ Sítio } & \multirow{2}{*}{ Equação } & \multicolumn{3}{|c|}{ Coeficientes } & \multicolumn{3}{|c|}{ Parâmetros } \\
\hline & & $\beta_{0}$ & $\beta_{1}$ & $\boldsymbol{R}_{a j}^{2}$ & $S_{y x}$ & $\mathrm{CV}$ & AIC \\
\hline S1 & \multirow{3}{*}{$\operatorname{Lnh}=\beta_{0}+\beta_{I} *\left(\frac{1}{\text { dap }}\right)$} & 3,0728 & $-11,2411$ & 0,4598 & 0,158 & 5,63 & $-289,4$ \\
\hline $\mathrm{S} 2$ & & 3,1788 & $-12,1550$ & 0,2592 & 0,185 & 6,38 & $-312,4$ \\
\hline S3 & & 3,1183 & $-10,6102$ & 0,4636 & 0,136 & 4,93 & $-305,7$ \\
\hline
\end{tabular}

Onde: Sítio 1 = Bom Jardim da Serra; Sítio 2 = Painel; Sítio 3 = São José do Cerrito; dap = diâmetro a 1,30 $\mathrm{m}$ de altura do solo $(\mathrm{cm}) ; \mathrm{h}=$ altura total $(\mathrm{m}) ; \beta_{0}$ e $\beta_{1}=$ coeficientes ajustados; $\mathrm{R}^{2}{ }_{\mathrm{aj}}=$ coeficiente de determinação ajustado; $\mathrm{S}_{\mathrm{yx}}=$ erro padrão da estimativa; $\mathrm{CV}=$ coeficiente de variação (\%); AIC $=$ critério de informação de Akaike.

Tabela 2. Parâmetros dendrométricos e morfométricos de Araucaria angustifolia em três sítios com altitudes distintas, em Santa Catarina, Brasil.

Table 2. Dendrometric and morphometric parameters of Araucaria angustifolia in three sites with different altitudes, in Santa Catarina State, Brazil.

\begin{tabular}{|c|c|c|c|c|c|c|c|c|c|c|}
\hline \multicolumn{11}{|c|}{$\mathrm{S} 1\left(\mathrm{~N}=362\right.$ árvores $\left.\mathrm{ha}^{-1}\right)$} \\
\hline & dap & $\mathbf{h}$ & hi & dc & ac & pc & is & ge & ia & fc \\
\hline Média & $32,63 a$ & $15,1 \mathrm{a}$ & $12,5 \mathrm{a}$ & $6,5 \mathrm{a}$ & 38,0 & 17,2 & 0,2 & $0,5 \mathrm{a}$ & 0,4 & 3,2 \\
\hline Sd. & 8,8 & 2,9 & 2,7 & 2,4 & 25,2 & 10,5 & 0,1 & 0,1 & 0,1 & 1,6 \\
\hline Mín. & 12,73 & 8,0 & 3,5 & 1,8 & 2,4 & 3,0 & 0,1 & 0,3 & 0,1 & 0,7 \\
\hline Máx. & 55,07 & 23,2 & 18,7 & 12,1 & 114,0 & 65 & 0,3 & 0,9 & 0,8 & 6,9 \\
\hline \multicolumn{11}{|c|}{$\mathrm{S2}\left(\mathrm{N}=232\right.$ árvores $\left.\mathrm{ha}^{-1}\right)$} \\
\hline Média & $49,99 \mathrm{~b}$ & $18,8 b$ & $13,5 \mathrm{a}$ & $9,7 \mathrm{~b}$ & 79,8 & 27,1 & 0,2 & $0,4 \mathrm{~b}$ & 0,5 & 2,4 \\
\hline Sd. & 13,7 & 3,8 & 2,3 & 2,8 & 43,4 & 10,6 & 0,04 & 0,1 & 0,2 & 1,6 \\
\hline Mín. & 17,83 & 9,2 & 8,2 & 1,8 & 2,4 & 2,7 & 0,1 & 0,2 & 0,1 & 0,3 \\
\hline Máx. & 88,17 & 27,7 & 21,0 & 16,7 & 217,7 & 49,2 & 0,3 & 1,0 & 1,0 & 10,1 \\
\hline \multicolumn{11}{|c|}{$\mathrm{S3}\left(\mathrm{N}=403\right.$ árvores $\left.\mathrm{ha}^{-1}\right)$} \\
\hline Média & $33,81 \mathrm{a}$ & $16,2 \mathrm{a}$ & $7,2 \mathrm{~b}$ & $6,6 a$ & 36,9 & 54,5 & 0,2 & $0,5 \mathrm{a}$ & 0,4 & 0,8 \\
\hline Sd. & 10,8 & 2,8 & 2,1 & 2,0 & 22,4 & 13,7 & 0,03 & 0,1 & 0,1 & 0,3 \\
\hline Mín. & 11,55 & 7,2 & 2,5 & 2,8 & 5,9 & 16,9 & 0,1 & 0,3 & 0,2 & 0,3 \\
\hline Máx. & 59,84 & 23,7 & 13,3 & 11,9 & 110,3 & 84,8 & 0,3 & 0,8 & 0,8 & 2,9 \\
\hline
\end{tabular}

Onde: S1 = Bom Jardim da Serra; S2 = Painel; S3 = São José do Cerrito; $\mathrm{N}$ = densidade do povoamento; Sd. = desvio padrão; Min. = mínimo; Máx. = máximo; dap = diâmetro a $1,30 \mathrm{~m}$ de altura do solo $(\mathrm{cm}) ; \mathrm{h}=$ altura total $(\mathrm{m}) ; \mathrm{hi}=$ altura de inserção da copa $(\mathrm{m}) ; \mathrm{dc}=$ diâmetro de copa $(\mathrm{m}) ;$ ac $=$ área de copa $\left(\mathrm{m}^{2}\right) ; \mathrm{pc}=$ proporção de copa $(\%) ;$ is = índice de saliência; ge = grau de esbeltez; ia = índice de abrangência; fc = formal de copa. Letras diferentes na coluna representam diferença significativa a $5 \%$ de probabilidade, pelo teste de Kruskal-Wallis. 
torno da média (61,05\%), superior ao sítio $2(39,11 \%)$ e sítio $3(25,14 \%)$. No Sítio 3, 46 árvores (o que representa $59,7 \%$ ) apresentaram pc superior a $50 \%$, indicando que as árvores neste sítio necessitam de maior porcentagem do tronco com copa para manter a vitalidade. Nos demais sítios a relação pc foi inferior a 50\% (99,4\% dos indivíduos) explicada, em parte, pela maior altura de inserção de copa (hi).

Grande parte das variáveis morfométricas e dendrométricas avaliadas nesse estudo apresentou correlação significativa com o dap (Tabela 3).

Tabela 3. Matriz de correlação de Pearson entre as variáveis morfométricas e dendrométricas de Araucaria angustifolia em três sítios com altitudes distintas, em Santa Catarina, Brasil.

Table 3. Pearson correlation matrix between morphometric and dendrometric variables of Araucaria angustifolia in three sites with different altitudes, in Santa Catarina State, Brazil.

\begin{tabular}{|c|c|c|c|c|c|c|c|c|c|c|c|}
\hline \multicolumn{12}{|c|}{ Sítio 1} \\
\hline & Média & Sd. & dap & h & hi & dc & pc & ac & fc & is & ge \\
\hline dap & 32,63 & 8,78 & - & & & & & & & & \\
\hline h & 15,09 & 2,94 & $0,63^{*}$ & - & & & & & & & \\
\hline hi & 12,47 & 2,70 & 0,43 & $0,84^{*}$ & - & & & & & & \\
\hline dc & 6,57 & 2,36 & $0,70^{*}$ & $0,48^{*}$ & 0,22 & - & & & & & \\
\hline pc & 17,22 & 10,54 & 0,25 & 0,08 & $-0,46^{*}$ & 0,38 & - & & & & \\
\hline ac & 37,98 & 25,17 & $0,66^{*}$ & 0,41 & 0,18 & $0,98 *$ & 0,33 & - & & & \\
\hline fc & 3,23 & 1,58 & $-0,08$ & $-0,22$ & 0,22 & $-0,11$ & $-0,75^{*}$ & $-0,07$ & - & & \\
\hline is & 0,20 & 0,06 & $-0,01$ & 0,12 & $-0,05$ & $0,64 *$ & 0,29 & $0,62 *$ & $-0,11$ & - & \\
\hline ge & 0,48 & 0,12 & $-0,72^{*}$ & $-0,03$ & 0,06 & $-0,46^{*}$ & $-0,18$ & $-0,44^{*}$ & $-0,15$ & 0,12 & - \\
\hline ia & 0,44 & 0,15 & $0,44^{*}$ & 0,004 & $-0,21$ & $0,85^{*}$ & 0,39 & $0,86^{*}$ & $-0,03$ & $0,65^{*}$ & $-0,47 *$ \\
\hline \multicolumn{12}{|c|}{ Sítio 2} \\
\hline dap & 49,99 & 13,66 & - & & & & & & & & \\
\hline h & 18,79 & 3,82 & 0,38 & - & & & & & & & \\
\hline hi & 13,47 & 2,34 & 0,10 & $0,71^{*}$ & - & & & & & & \\
\hline dc & 9,71 & 2,82 & $0,82 *$ & 0,20 & $-0,08$ & - & & & & & \\
\hline pc & 27,07 & 10,61 & $0,48 *$ & $0,58^{*}$ & $-0,15$ & $0,42 *$ & - & & & & \\
\hline ac & 79,83 & 43,41 & $0,81 *$ & 0,19 & $-0,06$ & $0,98 *$ & 0,38 & - & & & \\
\hline fc & 2,43 & 1,57 & $-0,28$ & $-0,63^{*}$ & $-0,15$ & $-0,08$ & $-0,77^{*}$ & $-0,06$ & - & & \\
\hline is & 0,20 & 0,04 & $-0,24$ & $-0,22$ & $-0,25$ & 0,21 & $-0,06$ & 0,16 & 0,25 & - & \\
\hline ge & 0,40 & 0,14 & $-0,72 *$ & 0,24 & 0,36 & $-0,66^{*}$ & $-0,14$ & $-0,60 *$ & $-0,04$ & 0,1 & - \\
\hline ia & 0,53 & 0,16 & $0,46^{*}$ & $-0,44^{*}$ & $-0,51^{*}$ & $0,75^{*}$ & 0,01 & $0,74 *$ & 0,32 & 0,32 & $-0,71 *$ \\
\hline \multicolumn{12}{|c|}{ Sítio 3} \\
\hline dap & 33,81 & 10,77 & - & & & & & & & & \\
\hline $\mathbf{h}$ & 16,19 & 2,84 & $0,64 *$ & - & & & & & & & \\
\hline hi & 7,22 & 2,14 & $-0,04$ & 0,25 & - & & & & & & \\
\hline dc & 6,6 & 1,95 & $0,87^{*}$ & $0,52 *$ & $-0,21$ & - & & & & & \\
\hline pc & 54,54 & 13,74 & 0,41 & 0,35 & $-0,81 *$ & $0,49 *$ & - & & & & \\
\hline ac & 36,91 & 22,39 & $0,84^{*}$ & $0,48^{*}$ & $-0,23$ & $0,98 *$ & $0,48^{*}$ & - & & & \\
\hline fc & 0,8 & 0,34 & 0,14 & $-0,31$ & 0,37 & 0,21 & $-0,56^{*}$ & 0,20 & - & & \\
\hline is & 0,2 & 0,03 & $-0,11$ & $-0,20$ & $-0,19$ & 0,21 & 0,05 & 0,22 & 0,22 & - & \\
\hline ge & 0,51 & 0,13 & $-0,81^{*}$ & $-0,16$ & 0,24 & $-0,74 *$ & $-0,33$ & $-0,70^{*}$ & $-0,34$ & 0,01 & - \\
\hline ia & 0,4 & 0,11 & $0,54 *$ & $-0,11$ & $-0,35$ & $0,76^{*}$ & 0,26 & $0,76^{*}$ & $0,49 *$ & 0,36 & $-0,71^{*}$ \\
\hline
\end{tabular}

Onde: * = significativo a 95\% de probabilidade; $\mathrm{Sd} .=$ desvio padrão; dap = diâmetro a 1,30 $\mathrm{m}$ de altura do solo $(\mathrm{cm}) ; \mathrm{h}=$ altura total $(\mathrm{m}) ; \mathrm{hi}=$ altura de inserção de copa $(\mathrm{m})$; dc = diâmetro de copa $(\mathrm{m}) ; \mathrm{pc}=$ proporção de copa; ac = área de projeção de copa $\left(\mathrm{m}^{2}\right)$; fc = formal de copa; is = índice de saliência; ge $=$ grau de esbeltez; ia = índice de abrangência. 
Nos sítios 1 e 3, verificou-se que, à medida que aumentou o dap, a altura total, o diâmetro de copa, a área de copa e o índice de abrangência também aumentaram, resultando em uma correlação positiva. A maior correlação com o dap foi verificada com a variável diâmetro de copa, sendo 0,70 para o sítio 1 e 0,87 para o sítio 3 .

Para o sítio 2, verificou-se correlação positiva entre o dap e o diâmetro de copa, proporção de copa, área de copa e o índice de abrangência. A maior correlação foi com o diâmetro de copa $(0,82)$.

O grau de esbeltez apresentou correlação negativa nos três sítios analisados, sendo os valores de $-0,72$ para os sítios 1 e 2, e -0,81 para o sítio 3 .

Com base na área de projeção de copa, calculouse a fração de ocupação que as copas representam na área, indicando como estariam as copas se elas fossem colocadas em uma superfície (Tabela 4).

Tabela 4. Fração de ocupação de copa considerando o total de classes diamétricas e considerando somente árvores de Araucaria angustifolia com dap $\geq 40 \mathrm{~cm}$, em três locais, em Santa Catarina, Brasil.

Table 4. Fraction canopy occupation considering the total diameter classes and considering only trees of Araucaria angustifolia with $\mathrm{dbh} \geq 40 \mathrm{~cm}$ in three sites in Santa Catarina State, Brazil.

\begin{tabular}{ccccc}
\hline & \multicolumn{2}{c}{ Todas as classes diamétricas } & \multicolumn{2}{c}{ Acima de 40 cm de dap } \\
\hline Sítio & N & Fração de ocupação & N & Fração de ocupação \\
\hline S1 & 362 & 1,37 & 33 & 0,2 \\
S2 & 232 & 1,85 & 115 & 1,4 \\
S3 & 403 & 1,49 & 48 & 0,3 \\
\hline
\end{tabular}

Onde: $\mathrm{N}$ = número de árvores ha ${ }^{-1}$; S1 = Bom Jardim da Serra; S2 = Painel; e S3 = São José do Cerrito.

No primeiro cálculo onde foram consideradas todas as classes diamétricas presentes nos sítios, encontrou-se, nas três áreas, frações de ocupação acima de 1, indicando que as copas ultrapassaram a área do hectare.

Quando se utilizou somente as árvores maduras (dap $\geq 40 \mathrm{~cm}$ ) para análise, o índice de ocupação reduziu nos sítios 1 e 3, porém manteve-se ainda elevado no sítio 2 . Podem-se observar na Figura 3 as áreas de projeção de copa, para cada local, de acordo com os agrupamentos por diâmetro/estrato.

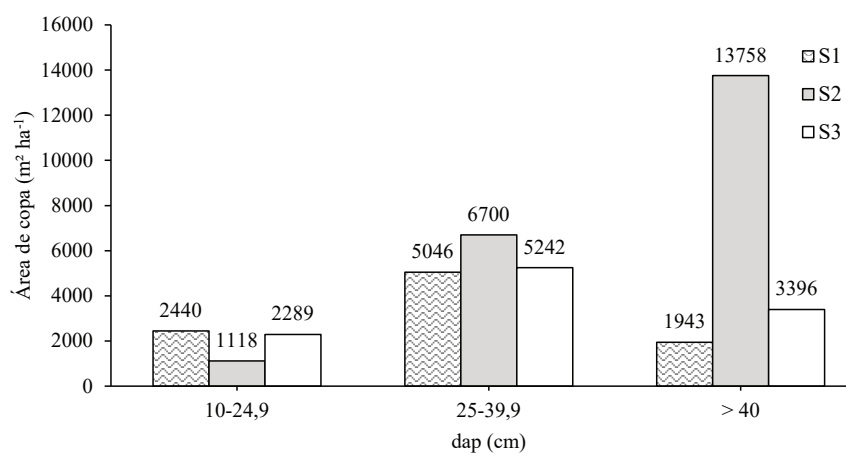

Figura 3. Área de projeção de copa por agrupamento e por diâmetro/estrato para Araucaria angustifolia em três sítios, em Santa Catarina, Brasil.

Figure 3. Projection area of canopy by diameter/stratum grouping for Araucaria angustifolia in three sites, in Santa Catarina State, Brazil.
Em relação às separações por estrato, para a obtenção dos dados de área de projeção de copa, para o sítio 2 o estrato superior alcançou, em relação aos estratos médio e inferior, respectivamente, valores aproximados de 12,3 e 2,05 vezes. Mesmo considerando os estratos inferior e médio somados, o estrato superior foi 1,76 vezes maior em projeção de área de copa.

\section{Discussão}

Apesar de algumas árvores apresentarem alturas semelhantes, podem estar em classes diamétricas diferentes, indicando maior investimento da árvore no componente altura. Reitz \& Klein (1966) afirmam que a araucária é uma espécie heliófila, porque que os indivíduos necessitam de luz para se desenvolverem. Assim, quando estão sofrendo o efeito da competição, acabam priorizando o crescimento em altura, para alcançar o índice luminoso de que precisam.

Segundo Zeide (1993), o crescimento resulta da interação de duas forças opostas. O componente positivo representa a tendência de qualquer ser vivo à multiplicação exponencial, estando este componente associado ao potencial biótico, atividade fotossintética, absorção de nutrientes, ao anabolismo etc. E o componente oposto representa as restrições ao crescimento impostas por fatores externos, tais como a 
competição, respiração, escassez de recursos naturais etc., e internos, como os mecanismos de auto regulação, conhecidos como resistência ambiental, metabolismo destrutivo, catabolismo etc.

As diferenças de altitude são fatores influentes sobre a estrutura florestal. Lawton (1982) verificou que o estresse provocado pelo vento faz com que as árvores dispendam mais energia com o seu suporte mecânico, às custas do crescimento em altura. No caso do sítio 1 desse estudo, é provável que a menor média de altura encontrada seja, em parte, uma resposta adaptativa ao estresse decorrido do vento presente em maior intensidade nesse sítio de maior altitude.

Além disso, a diversidade de espécies e a estatura da floresta diminuem com o aumento da altitude nas montanhas tropicais (Yamada, 1977; Ohsawa et al., 1985; Gentry, 1988; Proctor et al., 1988; Weaver \& Murphy, 1990; Nakashizuka et al., 1991; Lieberman et al., 1996).

Em relação ao índice de saliência (is), Araucaria angustifolia, em todas as altitudes estudadas, apresentou copa com aproximadamente 20 vezes o valor do diâmetro a 1,30 m do solo (dap). Por outro lado, apesar do sítio 2 apresentar média de diâmetro de copa (dc) superior, a relação de proporcionalidade dc/dap foi semelhante.

Roveda et al. (2012) reportaram índice similar para a relação dc/dap para um plantio de Araucaria angustifolia com 65 anos, quando o diâmetro de copa foi, em média, 23,7 vezes a dimensão do dap. Hess et al. (2016), estudando a espécie no Sul do Brasil, encontraram uma variação de 12,8 a 23,6 vezes o tamanho do dap para diferentes sítios e diâmetro de copa médio em torno de 20 vezes o dap. Klein et al. (2017), estudando morfometria de Araucaria angustifolia em São José do Cerrito no planalto catarinense, encontraram índice de saliência variando de 19,82 a 26,49 para árvores em diferentes posições sociológicas.

Para Sterba (1992), um menor valor no índice de saliência significa que a árvore possui, proporcionalmente, maior área de superfície de copa, sendo mais eficiente na utilização do espaço, considerando para isto uma dada área de projeção de copa. Assim, à medida que aumenta o diâmetro, a tendência do índice de saliência é diminuir, indicando a necessidade de desbaste do povoamento.

Segundo Brüchert \& Gardiner (2006), a mecânica das árvores é amplamente controlada pela forma externa da árvore, que é ajustada para equilibrar a estabilidade das árvores com a força do vento e os requisitos da árvore para fotossíntese. De acordo com esses autores, as árvores expostas ao vento, geralmente, apresentam menor porte, sendo mais afiladas em direção ao topo. Essa adaptação das árvores garante maior rigidez à flexão na base do caule devido ao maior diâmetro e maior flexibilidade na região da copa. A presença do vento em maior intensidade nas áreas de altitudes elevadas, como é o caso do S1, pode explicar, em parte, a menor proporção de copa encontrada, onde o alongamento dos galhos de algumas espécies pode ser retardado pela exposição ao vento (Lawton, 1982).

Outro fator presente na araucária é a desrama natural. Quando um galho é sombreado pela copa acima e pelas concorrentes vizinhas, ele reduz a produção fotossintética e logo é desconectado do sistema de abastecimento de assimilados (Nutto \& Spathelf, 2003). Sendo assim, árvores com grandes dap tendem a ter comprimentos de copa menores, quando comparados com outras espécies (Costa et al., 2016; Cisneros et al., 2019).

A observância do grau de esbeltez (ge) para árvores individuais revela que poucos indivíduos apresentaram ge $>0,5$, indicando que a maior parte das árvores está crescendo mais em diâmetro do que em altura.

O ge caracteriza a estabilidade das árvores. Quanto maior o ge, tanto mais instável é a árvore, ou mesmo servir como indicativo de desbastes atrasados, especialmente em povoamentos puros e equiâneos (Durlo \& Denardi, 1998).

Tonini \& Arco-Verde (2005) expõem que uma relação $\mathrm{h}$ /dap superior a 1,0 pode indicar a necessidade de desbaste, pois o crescimento em diâmetro é reduzido em relação à altura. Apesar do grau de ocupação ser elevado nos três sítios estudados, o valor da relação não se aproximou de 1 . Isso pode ser explicado pela estabilidade da altura máxima e pouca produtividade em diâmetro, indicando valores menores na relação hipsométrica.

À medida que a árvore fica mais velha, as diferenças entre a relação $\mathrm{h} /$ dap tendem a diminuir gradualmente. Isto ocorre porque após as árvores atingirem o seu clímax, a altura estabiliza, apesar do diâmetro continuar crescendo. Como resultado, as mudanças na relação h/ dap passam a ser gradualmente menos significativas (Finger, 1992).

O formal de copa (fc), expresso pela razão entre o diâmetro de copa e seu comprimento, variou de 0,8 (sítio 3) a 3,24 (sítio 1). Assmann (1970) expõe que o fc indica o grau de achatamento das copas das árvores ( $\mathrm{fc}<1=$ 
copas esbeltas; $\mathrm{fc}>1=$ copas achatadas) $\mathrm{e}$, quanto menor for esse índice, melhor será a produtividade da árvore. Portanto, constatou-se que $87 \%$ das árvores do sítio 3 possuíam $\mathrm{fc}<1$, indicando possivelmente árvores de maior produtividade e copas mais esbeltas, enquanto os sítios $1(98,7 \%)$ e $2(95,7 \%)$ tiveram a maior parte das árvores com copas mais achatadas ( $\mathrm{fc}>1)$.

Costa et al. (2016) relataram que o formal de copa de $A$. angustifolia é fortemente alterado com o decorrer do crescimento. Silva et al. (2017), pesquisando um plantio de $A$. angustifolia (40 anos), encontraram fc $>1$ para todas as árvores, afirmando ser uma característica comum em indivíduos adultos da espécie. Roveda et al. (2012) reportaram grande variabilidade de fc para A. angustifolia, com média de 2,15 , e afirmaram que o índice pode ser utilizado na seleção das árvores para desbaste, devendo ser retiradas aquelas com maior formal de copa. Um baixo valor de formal de copa significa que a árvore, para uma dada área de projeção de copa, tem disponível mais área de superfície de copa (área de assimilação) e, por isso, provavelmente ocupe o espaço mais eficientemente que uma árvore com maior formal de copa (Assmann, 1970).

Nos três sítios estudados as maiores correlações positivas foram observadas entre as variáveis diâmetro da copa e área da copa, e isto está relacionado à dependência entre as variáveis (Silva et al., 2017).

O diâmetro da árvore apresentou correlação negativa e superior a $70 \%$ com o ge, evidenciando que as árvores crescem mais em altura do que em diâmetro, nos três sítios, tendo relação com a estabilidade das árvores, a competição e o sítio (Hess et al., 2016). Silva et al. (2017), em um plantio de A. angustifolia com 40 anos, encontraram correlação de $-0,81$, entre dap e ge, mostrando que, com o aumento em diâmetro, o ge diminui e ocorre o aumento na estabilidade dos indivíduos.

A percentagem de copa (pc) apresentou correlação negativa com altura de inserção de copa (hi) para o sítio $1(-0,46)$ e sítio $3(-0,81)$. Klein et al. (2017), em estudo realizado com $A$. angustifolia, também encontraram correlação negativa entre a proporção de copa e hi $(-0,86)$, e indicaram que os valores discrepantes se referem a fatores genéticos, qualidade de sítio, grau de competição e condições biológicas e edafoclimáticas.

Nos três locais, o dap apresentou relação positiva com o diâmetro de copa $(\mathrm{dc})$, indicando que, à medida que a árvore aumenta em dap, o diâmetro de copa também aumenta. Da mesma forma, quanto maior foi o dc, maior foi a pc, para os sítios 2 e 3 . Para a relação dc e dap, Volkart (1969) encontrou coeficiente de correlação de 0,958 . À medida que as árvores crescem em dap, a dimensão de suas copas também aumenta, diminuindo, assim, o ge da árvore; ou seja, seu desenvolvimento é favorecido, garantindo sua posição no estrato dominante da floresta (Klein et al., 2017).

No sítio 2 a fração de ocupação de copa observada é elevada (Tabela 4). Em povoamentos fechados, as árvores dominadas com o tempo vão sendo sombreadas e podem ser cobertas pelas copas de suas árvores vizinhas. A ausência de árvores nas classes menores pode ser explicada pela fração de copa, que foi elevada na área, não permitindo a entrada de luz. Assim, com a ausência ou escassez de regeneração, aliada à mortalidade de árvores adultas, é possível que a população de $A$. angustifolia tenha um decréscimo do crescimento. Em áreas naturais, as copas muitas vezes estão em contato e sobrepostas umas às outras. Isso faz com que a área ocupada pelas copas represente quando somadas muito mais do que onde a floresta está inserida. No sítio 2, a fração de ocupação de copa é quase o dobro $(1,85)$, indicando que grande parte dessas copas estão sobrepostas. Essa grande ocupação pode comprometer a regeneração, possivelmente pela barreira enfrentada pela luz para penetrar no sub-bosque (Reitz \& Klein, 1966; Clark \& Clark, 1987; Duarte \& Dillenburg, 2000). Da mesma forma, quando se considera apenas os indivíduos adultos (dap $\geq 40 \mathrm{~cm}$ ), a área ainda apresenta uma fração de ocupação elevada (tabela 4), o que pode comprometer o crescimento, em decorrência da competição. $\mathrm{O}$ aumento progressivo da competição provoca uma redução no tamanho da copa e na eficiência dos ramos, o que resulta em declínios na produção de madeira (Larson, 1963).

Em relação às separações por estrato, para obtenção dos dados de área de projeção de copa por hectare, o sítio 2 apresentou valores elevados, o que indica dificuldade de regeneração nessa área,

O sítio 2 apresentou $64 \%$ do teto de cobertura. De acordo com os conceitos apresentados por Assmann (1970), a área florestal do sítio 2 estaria em estágio de produção completa ( $>50 \%$ do teto de cobertura), ou seja, a população já atingiu seu máximo desenvolvimento para área e está superestocada. Segundo o mesmo autor, os sítios 1 e 3 encontram-se em estágio de reestoqueamento. Entretanto, deve-se verificar se existe outra limitação 
ambiental para o estabelecimento e crescimento de $A$. angustifolia que possa estar influenciando a estrutura horizontal da espécie.

\section{Conclusões}

A menor média de altura encontrada no sítio $1(1.350 \mathrm{~m}$ de altitude) pode estar associada, em parte, com uma resposta adaptativa da espécie às condições de maior altitude do ambiente.

O sítio 2 (1.150 m de altitude) apresentou poucos indivíduos nas classes diamétricas iniciais, indicando deficiência de regeneração natural e, por conseguinte, um possível comprometimento no suprimento de Araucaria angustifolia para as maiores classes de diâmetro, no decorrer do tempo.

Nos sítios 1 e 3 (950 $\mathrm{m}$ de altitude) foi obtida correlação positiva do diâmetro a $1,30 \mathrm{~m}$ de altura do solo (dap) com altura total, diâmetro de copa, área de copa e índice de abrangência. No sítio 2 as correlações positivas do dap foram com o diâmetro de copa, proporção de copa, área de copa e índice de abrangência. As correlações de dap com o grau de esbeltez foram negativas para os três sítios.

A ausência de correlação do dap com altura no sítio 2 pode ser explicada pela influência das árvores do estrato superior. Após as árvores atingirem o clímax, o incremento em altura proporcionalmente sofre uma redução maior que o incremento em diâmetro.

\section{Agradecimentos}

O presente trabalho foi realizado com apoio da Coordenação de Aperfeiçoamento de Pessoal de Nível Superior - Brasil (CAPES) - Código de Financiamento 001.

\section{Referências}

Assmann, E. Principles of forest yield study. Oxford: Oxford Pergamon Press, 1970.

Braz, E. M. et al. Criteria to be considered to achieve a sustainable second cycle in Amazon Forest. Pesquisa Florestal Brasileira, v. 35, n. 83, p. 209-225, 2015. https://doi.org/10.4336/2015.pfb.35.83.941.

Brüchert, F. \& Gardiner, B. The effect of wind exposure on the tree aerial architecture and biomechanics of Sitka spruce (Picea sitchensis, Pinaceae). American Journal of Botany, v. 93, n. 10, p. 1512-1521, 2006. https://doi.org/10.3732/ajb.93.10.1512.
Chen, Q. et al. Isolating individual trees in a savanna Woodland using small footprint lidar data Photogramm. Photogrammetric Engineering \& Remote Sensing, v. 72, n. 8, p. 923-932, 2006. https://doi.org/10.14358/PERS.72.8.923.

Cisneros, A. B. et al. Morfometría de copa em Prosopis alba Griseb. Ciência Florestal, v. 29, n. 2, p. 1-22, 2019. https://doi. org/10.5902/1980509826846.

Clark, D. A. \& Clark, D. B. Análisis de la regeneración de árboles del dossel em bosque muy húmedo tropical: aspectos teóricos y prácticos. Revista de Biologia Tropical, v. 35, supl. 1, p. 41-54, 1987.

Costa, E. A. et al. Influência da posição social nas relações morfométricas de Araucaria angustifolia. Ciência Florestal, v. 26, n. 1, 2016. http://dx.doi.org/10.5902/1980509821116.

Daniel, T. W. et al. Principles of silviculture. New York: McGrawHill Book Company, 1979.

Duarte, L. S. \& Dillenburg, L. R. Ecophysiological responses of Araucaria angustifolia (Araucariaceae) seedlings to different irradiance levels. Australian Journal of Botany, v. 48, p. 531-537, 2000. https://doi.org/10.1071/BT98046.

Durlo, M. A. \& Denardi, L. Morfometria de Cabralea canjerana, em mata secundária do Rio Grande do Sul. Ciência Florestal, v. 8, n. 1, p. 55-66, 1998. http://dx.doi.org/10.5902/19805098351.

Epagri. Dados e informações biofísicas da Unidade de Planejamento Regional Planalto Sul Catarinense: UPR 3. Florianópolis: EPAGRI/CIRAM, 2002. 70 p.

Feldpausch, T. R. et al. Height-diameter allometry of tropical forest trees. Biogeosciences Discussions, v. 7, p. 7727-7793, 2011. https:// doi.org/10.5194/bg-8-1081-2011.

Finger, C. A. G. Fundamentos de biometria florestal. Santa Maria, RS: Ed. UFSM/CEPEF/FATEC, 1992. 269 p.

Gentry, A. H. Changes in plant community diversity and floristic composition on environmental and geographic gradients. Annals of the Missouri Botanical Garden, v. 75, n. 1, p. 1-34, 1988. https:// doi.org/10.2307/2399464.

Hess, A. F. et al. Morfometría de la copa de Araucaria angustifolia en sitios naturales en el sur de Brasil. Bosque (Valdivia), v. 37, n. 3, p. 603-611, 2016. http://dx.doi.org/10.4067/S071792002016000300017

Klein, D. R. et al. Relações morfométricas para Araucaria angustifolia (Bertol.) Kuntze em Santa Catarina. Floresta, v. 47, n. 4, p. 501-512, 2017. http://dx.doi.org/10.5380/rf.v47i4.49667.

Koch, B. et al. Detection of individual tree crowns in airborne lidar data Photogramm. Photogrammetric Engineering \& Remote Sensing, v. 72, n. 4, p. 357-363, 2006. https://doi.org/10.14358/ PERS.72.4.357.

Kwak, D. A. et al. Detection of individual trees and estimation of tree height using LiDAR data. Journal of Forest Research, v. 12, p. 425-434, 2007. https://doi.org/10.1007/s10310-007-0041-9.

Larson, P. R. Evaluating the environment for studies of the inheritance of wood properties. In: World consultation on forest and tree improvement, 1., 1963, Stockholm. Proceedings. Rome: FAO, 1963. p. 1-6. 
Lawton, R. O. Wind stress and elfin stature in a montane rain forest tree: an adaptive explanation. American Journal of Botany, v. 69, p. 1224-1230, 1982. https://doi.org/10.1002/j.1537-2197.1982. tb13367.x

Leite, P. F. \& Klein, R. M. Vegetação. In: Geografia do Brasil: Região Sul. v. 2. Rio de Janeiro: IBGE, 1990.

Lieberman, D. et al. Tropical forest structure and composition on a large-scale altitudinal gradient in Costa Rica. Journal of Ecology, v. 84, n. 2, p. 137-152, 1996. https://doi.org/10.2307/2261350.

Machado, S. A. et al. Comportamento da relação hipsométrica de Araucaria angustifolia no capão da Engenharia Florestal da UFPR. Pesquisa Florestal Brasileira, n. 56, p. 5-16, 2008. https://doi. org/10.4336/2012.pfb.56.5.

Mähler Junior, J. K. F. \& Larocca, J. F. Fitofisionomias, desmatamento e fragmentação da Floresta com Araucária. In: Fonseca, C. R. et al. (Ed.). Floresta com araucária: ecologia, conservação e desenvolvimento sustentável. Ribeirão Preto: Holos, 2009. p. 243-252.

Mattos, J. R. de. O pinheiro brasileiro. Florianópolis: Ed. da UFSC, 2011. $608 \mathrm{p}$.

Nakashizuka, T. et al. Altitudinal zonation of forest communities in Selangor, Peninsular Malaysia. Journal of Tropical Forest Science, v. 4, p.233-244, 1991.

Nutto, L. \& Spathelf, P. Modelagem da desrama natural de Araucaria angustifolia (Bert.) O. Ktze. Revista Florestal, v. 33, n. 3, p. 295309, 2003. http://dx.doi.org/10.5380/rf.v33i3.2263.

Ohsawa, M. et al. Altitudinal zonation of forest vegetation on Mount Kerinci, Sumatra: with comparison to zonation in the temperate region of east Asia. Journal of Tropical Ecology, v. 1, p. 193-216, 1985. https://doi.org/10.1017/S0266467400000286.

Padoin, V. \& Finger, C. A. Relações entre as dimensões da copa e a altura das árvores dominantes em povoamentos de Pinus taeda L. Ciência Florestal, v. 20, n. 1, p. 95-105, 2010. http://dx.doi. org/10.5902/198050981764.

Proctor, J. et al. Ecological studies on Gunung Silam, a small ultrabasic mountain in Sabah, Malaysia. I. Environment, forest structure and floristics. Journal of Ecology, v. 76, n. 2, p. 320-340, 1988. https://doi.org/10.2307/2260596.

Reitz, R. \& Klein, R. M. Araucariáceas. In: Reitz, R. Flora Ilustrada Catarinense. Itajaí: Herbário Barbosa Rodrigues, 1966. 62 p.
Roman, M. et al. Variáveis morfométricas e relações interdimensionais para Cordia trichotoma (Vell.). Ciência Florestal, v. 19, n. 4, p. 473480, 2009. http://dx.doi.org/10.5902/19805098901.

Roveda, M. et al. Morfometria de Araucaria angustifolia (Bertol.) Kuntze na floresta nacional de São Francisco de Paula-RS. In: CONGRESSO FLORESTAL PARANAENSE, 4., 2012, Curitiba. Anais... [Curitiba]: Malinovski Florestal, 2012.

Shimizu, J. Y. et al. Variabilidade genética em uma população remanescente de araucária no Parque Nacional do Iguaçu, Brasil. Boletim de Pesquisa Florestal, n. 41, p. 18-36, 2000.

Silva, F. A. et al. Caracterização de índices morfométricos para Araucaria angustifolia plantada na Região Norte do Rio Grande do Sul. Advances in Forestry Science, v. 4, n. 3, p. 143-146, 2017. http://dx.doi.org/10.34062/afs.v4i3.5111.

Soares, C. P. B. et al. Dendrometria e inventário florestal. 2. ed. Viçosa, MG: Ed. UFV, 2011. 272 p.

Souza, R. P. M. et al. Estrutura da comunidade arbórea e aspectos da regeneração natural de remanescentes florestais paulistas com araucária. In: Cardoso, E. J. B. N. \& Vasconcellos, R. L. F. (Ed.). Floresta com araucária: composição florística e biota do solo. Piracicaba: FEALQ, 2015. p. 89-132.

Sterba, H. Forstliche ertragslehre. Wien: Universitätfür Bodenkultur. Wien, 1992. $160 \mathrm{p}$.

Tonini, H. \& Arco-Verde, M. F. Morfologia da copa para avaliar o espaço vital de quatro espécies nativas da Amazônia. Pesquisa Agropecuária Brasileira, v. 40, n. 7, p. 633-638, 2005. http://dx.doi. org/10.1590/S0100-204X2005000700002.

Valério, Á. F. et al. Análise florística e estrutural do componente arbóreo de um fragmento de Floresta Ombrófila Mista em Clevelândia, Sudoeste do Paraná. Revista Acadêmica: Ciência Animal, v. 6, n. 2, 2017.

Volkart, C. M. Determinación de la relación diámetro copa: diámetro tronco en Araucaria angustifolia y Pinus elliottii en la Provincia de Misiones. In: CONGRESO FORESTAL ARGENTINO, 1., 1969, Buenos Aires. Actas... Buenos Aires: Servicio Nacional Forestal. 1969.

Weaver, P. L. \& Murphy, P. G. Forest structure and productivity in Puerto Rico's Luquillo mountains. Biotropica, v. 22, n. 1, p. 69-82, 1990. http://dx.doi.org/10.2307/2388721.

Yamada, I. Forest ecological studies of the montane forest of Mt. Pangrango, West Java. IV. Floristic composition along the altitude. South East Asian Studies, v. 15, n. 2, p. 226-254, 1977.

Zeide, B. Analysis of growth equations. Forest Science, v. 39, n. 3, p. 594-616, 1993. https://doi.org/10.1093/forestscience/39.3.594. 